\title{
Gerontology
}

\section{Urinary Urgency in the Elderly}

\author{
Jeannette M. Potts Christopher K. Payne \\ Vista Urology and Pelvic Pain Partners, San Jose, CA, USA
}

\author{
Keywords \\ Elderly · Urinary urgency $\cdot$ Epidemiology $\cdot$ Pathophysiology . \\ Treatment
}

\begin{abstract}
The last 2 decades have brought an explosion of research, new products, and general attention to the problem of urinary urgency, and yet patients continue to be plagued by this symptom - especially the elderly. What is it? What does it mean? How can we guide patients successfully through this challenge? This paper presents a review of current thinking about urgency relevant to the practicing clinician, including the epidemiology, pathophysiology, evaluation, and treatment of these patients.

(c) 2018 S. Karger AG, Basel
\end{abstract}

\section{Background/Terminology}

The terminology surrounding urgency remains somewhat controversial. At present, the word "urge" is used to describe the normal phenomenon of bladder sensations whereas "urgency" is used to refer to pathology. The current International Continence Society definitions [1] are:

- Urgency: "Urgency is a lower urinary tract (storage) symptom (LUTS) defined as the complaint of a sudden compelling desire to pass urine, which is difficult to defer."

\section{KARGER}

(c) 2018 S. Karger AG, Basel

E-Mail karger@karger.com

www.karger.com/ger
- Urgency (urinary) incontinence (UUI): Complaint of involuntary loss of urine associated with urgency.

- Overactive bladder $(O A B)$ syndrome: Urinary urgency, usually accompanied by frequency and nocturia, with or without urgency urinary incontinence, in the absence of urinary tract infection (UTI) or other obvious pathology.

Thus, urgency is the cardinal symptom of OAB and the two terms are unfortunately often used interchangeably. Before the introduction of the term OAB in 2002 there was a lack of appreciation for urgency as a symptom separate from UUI. Subsequent research has divided the $\mathrm{OAB}$ population into "OAB wet" (with associated UUI) and "OAB dry" (no incontinence).

These definitions work well for a very large spectrum of patients who present with complaints of lower urinary tract symptoms. However, it should be acknowledged that another significant group of patients complain mostly of a constant, bothersome urge which is not at all "sudden." This distinction parallels the two Oxford Dictionary definitions of urgency [2]:

1. Importance requiring swift action (parallels current urological use).

2. An earnest and persistent quality; insistence (not covered by common urological use).

When bladder pain is associated these patients are easily classified into bladder pain syndrome/interstitial cystitis. However, there remain many patients who do not endorse either pain or urgency as currently defined [3]. This review will focus only on classic urgency/OAB.

Christopher K. Payne, MD

Emeritus Professor of Urology, Stanford University Co-Founder, Vista Urology and Pelvic Pain Partners 2998 South Bascom Avenue, Suite 100, San Jose, CA 95124 (USA)

E-Mail drpayne@vistaurology.com 
Table 1. Prevalence (\%) of urgency and OAB: large population-based studies

\begin{tabular}{|c|c|c|c|c|c|c|c|c|c|}
\hline $\begin{array}{l}\text { First } \\
\text { author }\end{array}$ & Year & $\begin{array}{l}\text { Age, } \\
\text { years }\end{array}$ & \multicolumn{3}{|l|}{ Men } & \multicolumn{3}{|l|}{ Women } & Criteria \\
\hline $\begin{array}{l}\text { Irvin } \\
{[6]}\end{array}$ & 2006 & $\geq 18$ & 10.8 & 1.2 & 9.6 & 12.8 & 6.3 & 6.5 & Urgency must be present \\
\hline
\end{tabular}

$\mathrm{OAB}$, overactive bladder.

\section{Epidemiology}

There is no doubt that urgency and the OAB syndrome are prevalent and bothersome. Several large populationbased studies have investigated the prevalence of urgency and $\mathrm{OAB}$ in adults [4-6]. Although each study used somewhat different definitions and procedures, and covered different regions of Europe and North America, all found high prevalence rates (ranging from 10.8 to 17.4\%) for both men and women (see Table 1). The influence of race and ethnicity is less well studied but the research that has been done suggests that all groups appear to be significantly affected.

$\mathrm{OAB} /$ urgency is not only prevalent, it is bothersome to patients. It appears that the frequency and severity of urgency is the key driver of patient bother; in fact, a highquality study identified urgency as the most prevalent bothersome lower urinary tract symptom for the population as a whole at 7.9\% [7]. The presence of UUI increases the impact compared to "OAB dry" but even patients without incontinence can suffer significant negative effects on activities, work, and relationships despite employing a variety of coping strategies. The overall economic [8] and personal [9] burdens of UUI have been reviewed.

All studies show similar and markedly increasing prevalence of $\mathrm{OAB}$ with age for both men and women (Fig. 1) The main difference is that women develop incontinence $(\mathrm{OAB}$ wet) at a much earlier age, with men lagging by several decades. The aging phenomenon has often been attributed to muscular or neurological changes occurring in the "aging bladder." However, there is data challenging the idea that OAB might be a "normal" part of aging.
McGrother et al. [10] used a postal survey of a random sampling of over 19,000 community dwelling women $>40$ years of age in the UK with 1-year follow-up surveys. They found a strong correlation between poorer health and increased prevalence of OAB. In analyzing this they concluded that "OAB was independently predicted by poor health. The association with old age, although consistent with other studies, disappeared after controlling for a full range of specific comorbidities, suggesting that the condition is age related rather than age dependent." Suskind et al. [11] examined this issue in a different way. Her group studied older individuals in a urologic practice by having them perform a Timed Up and Go Test (TUGT). Over 1,300 patients $\geq 65$ years were tested and the results correlated with the urologic diagnosis. They found that frailty, as defined by a slow time on the TUGT, "when adjusted for age, race, and gender, is a statistically significant predictor of $O A B$ " $(\mathrm{OR}=3.0)$. Those older individuals with "fast" times on TUGT (normal) had only a $10.6 \%$ rate of $\mathrm{OAB}$, similar to or lower than the population at large.

Thus, it is reasonable to expect that healthy older individuals should have normal bladder function. As we will discuss in the following sections, there are many ways in which the common comorbidities of aging may cause the symptoms of $\mathrm{OAB}$.

\section{Pathophysiology}

In discussing the pathophysiology of urgency it is important to consider:

- Neurogenic bladder (NGB) versus OAB. 


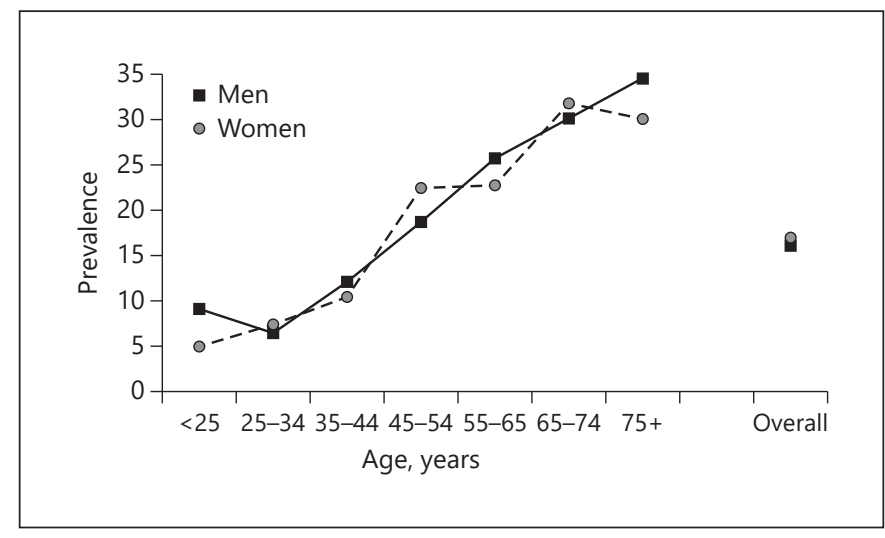

Fig. 1. Prevalence of overactive bladder with age for both men and women.

- Theorized etiology of OAB.

- Identifiable, correctable causes of OAB syndrome. $N G B$ versus $O A B$. Neurogenic bladder is defined by the International Continence Society as "abnormal or difficult function of the bladder, urethra (and/or prostate in men) in mature individuals in the context of clinically confirmed relevant neurologic disorder" [12]. Some clinical reports use the terminology neurogenic- and idiopathic-OAB. This is not preferred. OAB is properly considered to be an idiopathic condition "in the absence of obvious pathology." However, this distinction is not always easy to apply - e.g., which diabetic patients with urgency should be considered to have NGB?

Theorized Etiology of $O A B$. It is important to realize that $\mathrm{OAB}$ is a syndrome, not a disease. We know neither the etiology nor the underlying pathophysiology. As discussed in more detail below, it was long assumed that the experience of urgency represented the occurrence of an abnormal bladder contraction. It is clear that this is frequently not the case but research (and thinking) is still driven by the search for a cause for abnormal bladder contractions. It is much more difficult to study bladder sensation and much remains unknown in this regard. It is likely that the problem of urgency and the OAB syndrome represent the clinical endpoint of a variety of different initiating causes (and that some patients may have more than one cause). Some proposed etiologies of OAB are:

- Myogenic: Proposed by Brading [13] based on observations that changes in detrusor myocytes lead to increased excitability and increased ability of activity to spread between cells, thus propagating focal abnormal bladder contractions, and that these alterations were a "necessary prerequisite" for bladder overactivity of any etiology.

- Neurogenic: Proposed by de Groat [14] suggesting that subclinical neurological changes (either sensitization of afferents to the bladder or diminished central inhibition) produce detrusor overactivity.

- Urethrogenic: Proposes that urethral instability (sudden loss of urethral pressure during filling) and/or loss of the tonic inhibitory effect of the urethral sphincter is the cause of bladder overactivity. This idea is supported by observations that women with bladder overactivity have lower urethral muscle mass than agematched controls [15] and that urethral muscle density declines with aging [16].

- Ischemic: Animal models for ischemia-induced detrusor overactivity exist. The increasing prevalence of $\mathrm{OAB}$ with age could be due to the associated increase in atherosclerotic disease [17].

- Obstruction: Partial obstruction is a common animal model for detrusor overactivity. Morphological changes in the bladder are often not reversed when obstruction is relieved (e.g., when a man has a prostatectomy) and storage symptoms often persist.

- Mucosa: In the past the bladder epithelium was viewed as a simple barrier. A great deal of research in the past decade has elucidated relevant epithelial physiological and sensory functions that could have important impacts on bladder function, including producing detrusor overactivity [18].

Identifiable, Correctable Causes of OAB Syndrome. It is important to remember that the definition of $\mathrm{OAB}$ includes the qualification, "in the absence of urinary tract infection (UTI) or other obvious pathology." There are many specific, correctable causes of urgency/OAB in addition to those properly classified as NGB discussed above.

- Bladder outlet obstruction: Storage symptoms may result from obstruction of the urine outflow, commonly due to prostate enlargement. This phenomenon occurs secondary to bladder wall changes in response to increased bladder outlet resistance - detrusor hypertrophy, collagen deposition, decreasing bladder wall compliance, and acquired neurogenic changes. Other causes of bladder outlet obstruction include strictures of the urethra, rare neoplasms of the urethra, stones or foreign body, or neurogenic disease promoting detrusor sphincter dyssynergia.

- Polyuria: The patient may simply be producing too much urine and have normal bladder function. One common example is in the setting of diuresis second- 
Table 2. Causes of nocturnal polyuria

- Chronic heart failure

- Diabetes mellitus (uncontrolled)

- Diabetes insipidus

- Hypoalbuminemia

- Sleep apnea

- Multiple sclerosis

- Depression

- Renal disease

ary to hyperglycemia in poorly controlled or undiagnosed diabetes. Polydipsia is a rarer cause and is usually associated with certain medications or psychological disorders. A fluid diary will elucidate this diagnosis.

- Nocturia: Symptoms occurring only at night are more likely due to nocturnal polyuria. All too often, patients presenting with nocturia are diagnosed and treated for presumed urological conditions (especially $\mathrm{BPH}$ in men). In fact, over $88 \%$ of nocturia is due to nocturnal polyuria [19], which has several serious medical nonurological causes (see Table 2). Nocturnal polyuria is easily confirmed with a fluid diary. See Box 1 to learn how to calculate the Nocturnal Polyuria Index (NPi) and interpret thresholds.

- Metabolic syndrome (MetS) and obesity: MetS and obesity are strongly correlated with LUTS in men and women - especially storage symptoms. Although some studies demonstrate an association with benign prostate volume and MetS, the lower urinary tract symptoms best correlated with MetS are those of storage [20]. Most obesity studies have examined morbidly obese patients with an endpoint of overall urinary incontinence; the strongest effect is on stress urinary incontinence (SUI). However, there is increasing evidence that even patients with more moderate degrees of obesity are affected and even modest weight loss can improve all types of incontinence [21].

- Cystocele/pelvic organ prolapse: Traction on the bladder with change in position and/or activity can stimulate urgency in women. In this case urgency will not occur at rest and is relieved when the prolapse is corrected with a pessary or surgery.

- Stress urinary incontinence: As with prolapse discussed above, urgency is stimulated by physical activity and does not occur at rest. This can occur in women (mostly after childbirth and pelvic surgery) and men (after prostatectomy).
Box 1.

The Nocturnal Polyuria Index (NPi)

Fluid diary: Whenever possible, provide the patient with a measuring device, graduate, or urinal. Instruct the patient to document the hour of the day or night along with the corresponding volume of urination. He should also indicate the time he goes to bed and the time he awakens to begin his day.

Add the sum of exactly $24 \mathrm{~h}$ of urination, being sure to capture a complete period of sleeping hours including the very first voided volume upon awakening. This is the denominator of the NPi.

Now add up the volume of urination beginning with the first one after retiring for bed and ending with the volume excreted upon awakening to begin the day. This total represents all the urine "produced" after retiring for sleep, and this sum is the numerator of the NPi.

Nocturnal volume/total 24 -h volume $=\mathrm{NPi}$.

Diagnosis of nocturnal polyuria in persons $<65$ years, when $\mathrm{NPi}>0.25$.

Diagnosis of nocturnal polyuria in persons $>65$ years when $\mathrm{NPi}>0.33$.

If polydipsia is suspected, include intake with output diary.

- Atrophic vaginitis: Atrophic vaginitis is commonly associated with nonspecific lower urinary tract symptoms including urgency; these are often reversible with replacement therapy.

\section{Evaluation}

\section{History}

The health care provider must understand both the primary symptom and any related bladder issues. The interview must be conducted in a manner similar to pain complaints:

- How often does the patient experience urinary urgency (and how often does the patient actually use the toilet) during both the day and night?

- How severe is the urgency? Does the patient experience incontinence and/or interrupt activities?

- Are there associated problems with emptying - hesitancy, weak stream, straining, intermittency?

- Are there exacerbating and ameliorating factors?

It is important to enquire about the past medical history bearing in mind the medical conditions affecting bladder function listed in Table 3. 
Table 3. Conditions associated with urinary urgency and UUI

- Diabetes
- Obesity
- Metabolic syndrome
- Multiple sclerosis
- Parkinson's disease
- Stroke
- Spinal injury
- Depression
- Hypertension
- Irritable bowel syndrome
- Fecal impaction
- Pelvic surgery
- Spinal surgery

UUI, urgency (urinary) incontinence.

In the review of systems, hormonal status, sexual functioning, neurosensory changes, gastrointestinal function, and bowel habits are assessed. Bowel function is strongly correlated with urinary symptoms and must also be taken into account when formulating a treatment strategy. A list of medications should not only include dosages, but should be documented as a time line so as to explore commonly associated pharmacological causes of urinary adverse effects.

\section{Physical Examination}

The first part of the examination is observing the patient's ability to transfer and ambulate. Functional limitations are an important contributor to the severity of OAB. Neurological evaluation to determine strength and/or sensory deficits can provide clues to causes or contributors of $\mathrm{OAB}$. If the patient is examined immediately after voiding, during the physical examination, a catheter or ultrasound can be used to confirm adequate bladder emptying when clinically indicated.

After a standard abdominal examination, the pelvic floor is evaluated. Inspection for signs of urinary and/or fecal incontinence should be performed: integrity of the perineal skin, intragluteal folds, and perianal health. In women, estrogen status would be determined. After inspection, sensory testing of the saddle region is carried out.

Pelvic floor tone is determined via digital vaginal and/ or rectal examination (high, normal, or low/absent). Recruitment of pelvic floor muscles is tested by asking the patient to contract the sphincter and relax. Laycock developed the modified Oxford Grading System to evaluate the strength of the pelvic floor muscles by using vaginal palpation. It consists of a 6 -point scale: $0=$ no contraction, $1=$ flicker, $2=$ weak, $3=\operatorname{moderate}, 4=\operatorname{good}($ with lift), and $5=$ strong [22]. Perineal excursion is observed during these maneuvers as well as the ability to isolate only the pelvic muscles without supplementing with thighs, abdominals etc. The examiner must be very careful in assessing the recruitment of sphincteric muscles not only during uptake/contraction, but also during relaxation. A poor contraction may be misinterpreted, for example, as weakness when in fact it may indicate abnormally high resting tone with muscle fatigue or an inability to further recruit muscle contraction.

Urinary incontinence has usually been associated with elongated/weak pelvic muscles. However, from the realm of physical medicine and myofascial pain syndromes, we have learned that overworked and shortened muscles of the pelvic floor and even the abdominal wall can cause a sensation of constant urinary urgency (particularly in younger patients). Therefore, pelvic floor hypertonus must be considered in the differential diagnosis of $\mathrm{OAB}$. Both men and women may suffer from increased urinary urgency and frequency, with or without pain, as a result of increased pelvic floor muscle tone.

In women, a careful bimanual examination via the vagina is done to rule out any masses. The patient is asked to bear down to assess prolapse. Any part of the vagina protruding beyond the hymen suggests clinically relevant prolapse. The authors strongly advocate digital rectal examination in all patients to assess for sphincteric tone, masses, anorectal pathology, presence of feces in rectal vault, fecal impaction, etc.

In men, we also recommend a lithotomy position to facilitate adequate inspection of the saddle area. A similar evaluation is carried out. In addition to possible findings on digital rectal examination, listed above, the prostate is palpated for both size and texture.

Urinalysis completes the physical examination. In the setting of OAB symptoms, one may find an abnormal white or red blood cell count, which can herald the presence of infection, inflammation due to ureteral or bladder calculi, or neoplasm. Low specific gravity may indicate polyuria due to renal or endocrine issues, or overhydration. Proteinuria can also be indicative of renal disorders impacting urine dilution. Glycosuria may indicate new onset or poorly controlled diabetes (but even with normal glycemic control, patients can suffer from diabetic cystopathies, some of which manifest with OAB symptoms). When hematuria cannot be explained by infection and corrected after adequate therapy, imaging studies and cystoscopy are indicated. 
This is a great deal to accomplish in a typical office visit. It is often convenient to briefly review what has been found and instruct the patient to complete a bladder diary and then return to review this and discuss treatment options when the full data set is available.

No additional testing is indicated for the average $\mathrm{OAB}$ patient. In our practice, additional evaluation is carried out when:

- The patient has "complicated OAB" (pain, neurological disorder, diagnosis unclear after initial workup, etc.).

- There is abnormal urine sediment, pyuria or hematuria, indicating ongoing infection, inflammation, ureteral or bladder calculus, or possible neoplasm. In these instances, imaging and cystoscopy are necessary.

- The patient does not respond to standard therapy.

Urological testing can include imaging, cystoscopy, and urodynamics. Full urodynamic studies are performed much less commonly than in the past. The primary indication for urodynamics is to look for other correctable abnormalities (obstruction, stress incontinence, poor bladder contractility), although some specialists feel that the severity and type of detrusor overactivity is relevant to counseling. In patients who have developed LUTS following pelvic or spinal surgery, urodynamics may be necessary as part of preliminary testing. Cystoscopy can identify specific causes of OAB (bladder cancer, carcinoma in situ, bladder stones, specific types of cystitis). Imaging is not part of the workup of $\mathrm{OAB}$ but can identify other urologic diseases (as mentioned above) or pelvic disorders that might cause OAB.

\section{Treatment}

Lower urinary tract symptoms do not present an immediate threat to health. Therefore, the therapy should stem from a joint process between the patient and clinician and consider the patient's goals/preferences. The Society of Urodynamics, Female Pelvic Medicine, and Urogenital Reconstruction (SUFU) has published excellent clinical care pathways on the website (http://sufuorg. com/resources/overactive-bladder-ccp.aspx). Therapy is divided into three levels - behavioral, pharmacological, and advanced therapies.

\section{First Line: Behavioral}

Behavioral therapy begins with education - education about anatomy, physiology, and the specifics of the patient's individual condition (Fig. 2). This starts with a

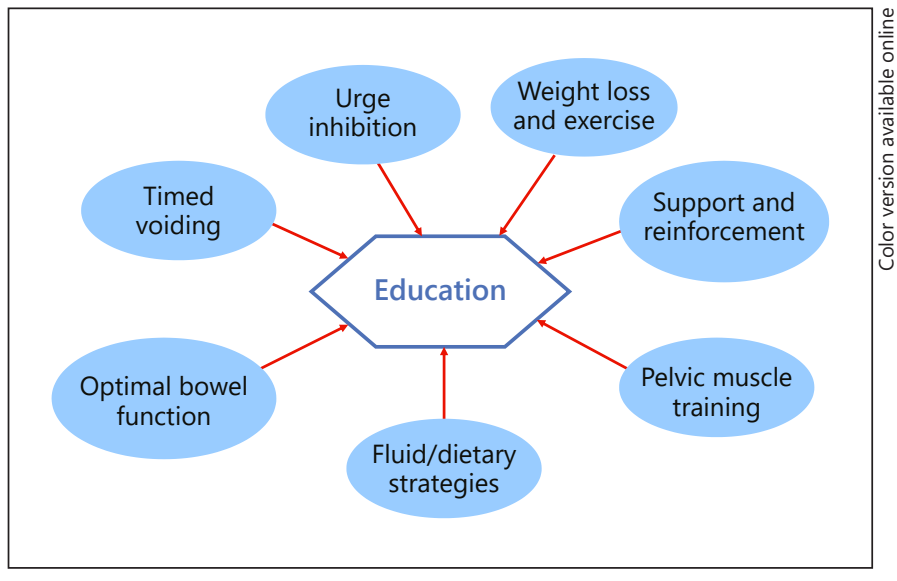

Fig. 2. First-line therapy for overactive bladder: behavioral (education).

bladder diary. Patients acquire insights into their condition and potential management strategies just by creating their bladder diary. Together with anatomical cartoons, the diary can be used to teach patients about normal bladder function and capacity (and used for goal setting). They are informed about appropriate hydration as well as the avoidance or restriction of irritating foods or beverages. The dosing and scheduling of medications affecting bladder function are adjusted if needed. Four strategies can be employed to regulate bladder function in different groups of patients - bladder training, timed voiding, prompted voiding, and toileting. All depend on the use of the bladder diary. Optimizing bowel function is paramount. When fecal urgency and frequency are associated with LUTS, addressing the gastrointestinal causes may also lead to resolution of OAB.

Interventions to strengthen pelvic muscles (pelvic floor muscle training) have long been recommended as first line therapy for SUI. Pelvic floor muscle training can also be useful in treating UUI and OAB, although recent Cochrane reviews indicate that research and evidence on pelvic training for UUI are limited and of low quality [23, 24].

Urge suppression teaches patients how to avoid bathroom (and other) cues that stimulate urgency. Patients are encouraged to pause, sit down if possible, relax the entire body, and contract pelvic floor muscles repeatedly to diminish urgency, inhibit detrusor contraction, and prevent urine loss. Only after the urgency subsides are they to proceed to the toilet at a normal pace [25]. Gradually, patients learn to suppress urgency by practicing these techniques and being more aware of their pelvic floor muscle tone [26]. 
$\mathrm{OAB}$ in men or women can also arise from shortened or hypertonic muscles of the pelvic floor; in these patients, pelvic muscle relaxation would be the focus of therapy. Therapy is most successful with training from a specialized professional. As recommended by The International Consultation on Incontinence, "clinicians should provide the most intensive health professional (HP) led pelvic floor muscle training program possible within service constraints, because HP taught and supervised programs are better than self-directed programs, and more HP contact is better than less" [27].

Weight loss and exercise are important means of treating OAB. Recent studies have demonstrated a correlation between a sedentary lifestyle and increased risk of urinary incontinence in women [28]. Similarly, obesity is strongly correlated with pelvic floor disorders, including urinary incontinence. Patients with lifelong physical activity have significantly lower risk of UI.

\section{Second Line: Pharmacological}

The 6th International Consultation on Incontinence gives nine oral drugs a Grade A recommendation for effectiveness based on Level 1 evidence: darifenacin, fesoterodine, imidafenacin, propiverine, solifenacin, tolterodine, trospium, oxybutynin, and mirabegron [29]. For practical purposes all of these can be considered to be standard antimuscarinic agents with the exception of mirabegron, which is a $\beta-3$ agonist. Treatment choice among these agents is often propelled by formulary decisions/ cost. Individuals may certainly do better with one than another but there is little evidence to suggest that any one medication is superior. All have demonstrable efficacy in high-quality trials. All have the same general range of antimuscarinic side effects with dry mouth the most commonly limiting use.

There is accumulating evidence that mirabegron has about the same degree of effectiveness as a standard antimuscarinic $[30,31]$ and that combination therapy with an antimuscarinic can produce improved control of symptoms [32]. Concerns about cardiovascular toxicity have largely proven to be unfounded [33]. Because of serious concerns about anticholinergics causing dementia and impaired cognition, mirabegron may have an increased role in treating $\mathrm{OAB}$ in the elderly.

\section{Third Line: Advanced Therapies}

Unfortunately, despite advances in pharmacological therapy, many patients remain dissatisfied with conservative measures. Some will want to move on to more invasive treatment (and perhaps many more would benefit from doing so). Fortunately, significant advances have been made in this arena as well. As these treatments typically require referral to a specialist, only a brief overview of each of the three major treatment options will be provided here; a review provides a more detailed analysis [34].

- Neuromodulation: Neuromodulation therapies are incompletely understood but clearly involve activation of afferent inhibitory pathways. Studies show changes in cortical activity of areas involved in lower urinary tract function with neuromodulation.

- Peripheral stimulation: Posterior tibial nerve stimulation has the strongest evidence base but vaginal, anal, and cutaneous stimulation have also been used. It is delivered in an office setting with stimulation of the nerve through an acupuncture type needle for $30 \mathrm{~min}$ weekly $\times 12$ weeks. It has been proven to be superior to sham therapy [35], to have equal efficacy and better tolerability than antimuscarinics (tolterodine) [36], and to have reasonably sustained long-term effect with maintenance therapy [37].

- Sacral nerve stimulation (SNS): Approved by the FDA for the treatment of urinary urgency incontinence in 1997 and subsequently for urgency-frequency without incontinence in 1999, SNS has been the mainstay of treatment for $\mathrm{OAB}$ patients who are refractory to pharmacological therapy. It can be effective for even severe and complex cases. A device is permanently implanted. There is a test phase which can be performed in the office with a temporary external wire or in the operating room with an implanted electrode. In either case the patient has an opportunity to evaluate the response before going on to the permanent generator implant. Surgery is generally performed under light sedation.

Approximately $2 / 3$ of patients with refractory $\mathrm{OAB}$ respond to SNS. Intermediate-term results are good with one study showing mean 4 -year responses of $70 \%$ for urgency incontinence and 68\% for urgency-frequency [38]. SNS should be considered for patients with refractory $\mathrm{OAB}$ and reasonable life expectancy who are healthy enough to tolerate a surgical procedure. Because SNS is also specifically approved for fecal incontinence and clearly effective for chronic constipation, it is typically preferred when these comorbidities are present.

- Botulinum toxin: Botulinum toxin binds to nerves using acetylcholine as a transmitter. On uptake into the cell, key proteins are cleaved, preventing the release of acetylcholine leading to muscle paralysis. The effect is reversed only with synthesis of new proteins and regrowth of axons. 


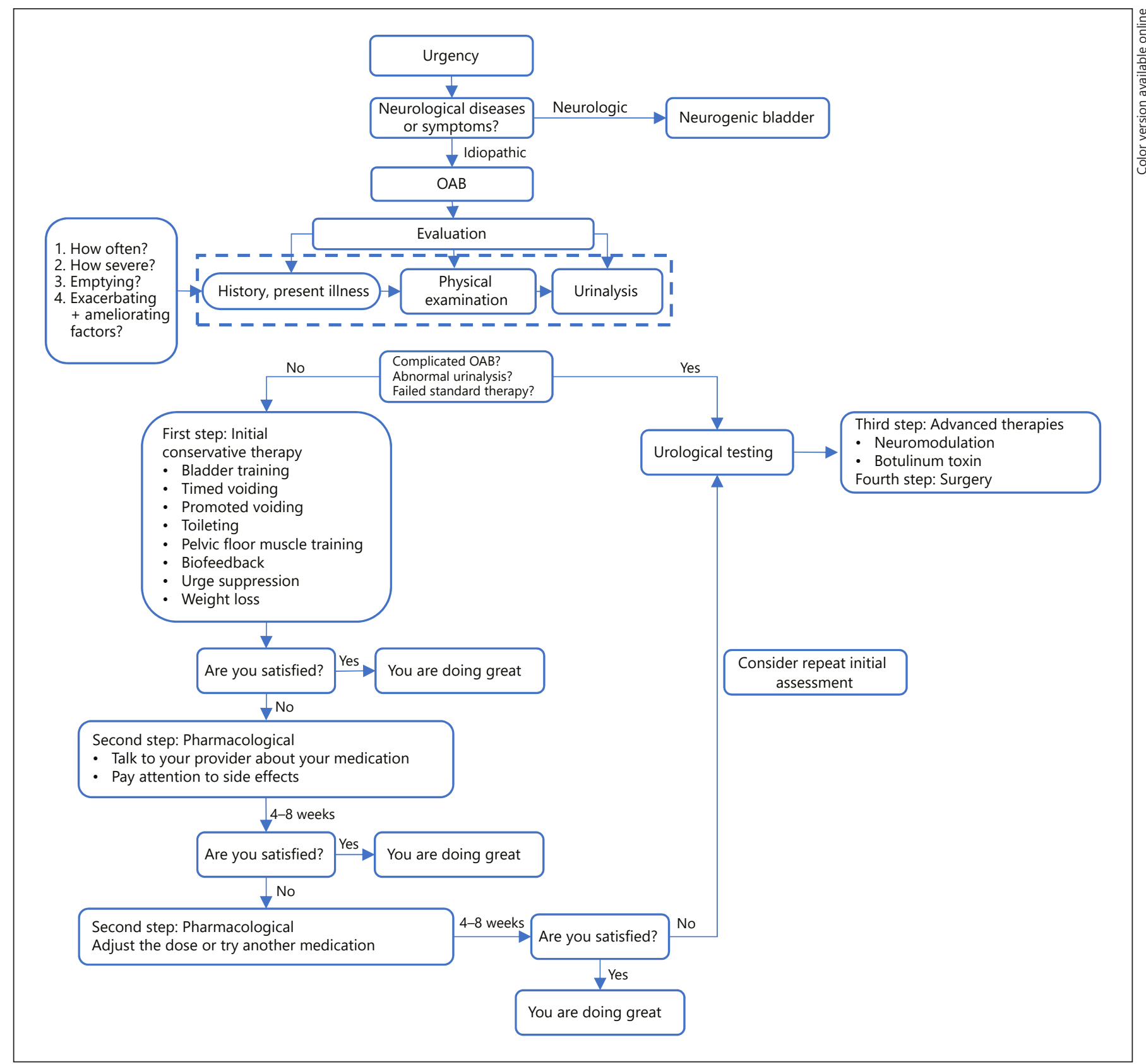

Fig. 3. Urinary urgency flowchart, including the three levels of therapy for overactive bladder (OAB): behavioral, pharmacological, and advanced therapies.

Botulinum toxin therapy requires cystoscopy and injection of the drug into the detrusor. This can usually be performed in the office under local anesthesia or light sedation. The procedure must be repeated over time (average duration in trials approximately 6 months). The efficacy appears to be at least similar to SNS [39] and superior to pharmacological therapy [40].

\section{Fourth Line: Diversion/Reconstruction}

The advances discussed above should mean that major surgical reconstruction (bladder augmentation, urinary diversion) and chronic catheter use should be rare occurrences. There are some neurogenic bladder patients with such severe spasticity that surgery is still required. Sadly, there remain many severely impaired patients, particu- 
larly elderly, in whom catheter drainage appears to be the only reasonable choice. A flowchart of the three levels of therapy for OAB (behavioral, pharmacological, and advanced therapies) is presented in Figure 3.

\section{Conclusion}

Urinary urgency is the hallmark of the OAB syndrome. It is prevalent in all age groups, men and women, and all races and ethnicities that have been studied. It often causes severe impact on quality of life. The pathophysiology is incompletely elucidated but successful evaluation and management are within the scope of most primary care physicians. When conservative measures are unsuccessful, advanced therapies such as neuromodulation and botulinum toxin injections can be employed to restore continence and quality of life for most patients.

\section{Disclosure Statement}

Dr. Potts' disclosures include consulting with Aquinox Pharma and Avadel Pharma. Dr. Payne's disclosures include consulting with Astellas, Aquinox, and Myoforte. The authors do not believe that these relationships create any conflict of interest related to this paper.

\section{References}

1 Haylen BT, de Ridder D, Freeman RM, et al: An International Urogynecological Association (IUGA)/International Continence Society (ICS) joint report on the terminology for female pelvic floor dysfunction. Int Urogynecol J 2010;21:5-26.

2 https://en.oxforddictionaries.com/definition/urgency.

3 Elliott CS, Payne CK: Interstitial cystitis and the overlap with overactive bladder. Curr Urol Rep 2012;13:319-326.

4 Stewart WF, Van Rooyen JB, Cundiff GW, et al: Prevalence and burden of overactive bladder in the United States. World J Urol 2003; 20:327-336.

5 Milsom I, Abrams P, Cardozo L, Roberts RG, Thuroff J, Wein AJ: How widespread are the symptoms of an overactive bladder and how are they managed? A population-based prevalence study. BJU Int 2001;87:760-766.

6 Irwin DE, Milsom I, Hunskaar S, et al: Population-based survey of urinary incontinence, overactive bladder, and other lower urinary tract symptoms in five countries: results of the EPIC study. Eur Urol 2006;50:1306-1315.

7 Agarwal A, Eryuzlu LN, Cartwright R, et al: What is the most bothersome lower urinary tract symptom? Individual- and populationlevel perspectives for both men and women. Eur Urol 2014;65:1211-1217.

8 Coyne KS, Wein A, Nicholson S, Kvasz M, Chen CI, Milsom I: Economic burden of urgency urinary incontinence in the United States: a systematic review. J Manag Care Pharm 2014;20:130-140.

9 Coyne KS, Wein A, Nicholson S, Kvasz M, Chen CI, Milsom I: Comorbidities and personal burden of urgency urinary incontinence: a systematic review. Int J Clin Pract 2013;67:1015-1033.
10 McGrother CW, Donaldson MM, Hayward $\mathrm{T}$, et al: Urinary storage symptoms and comorbidities: a prospective population cohort study in middle-aged and older women. Age Ageing 2006;35:16-24.

11 Suskind AM, Quanstrom K, Zhao S, et al: Overactive bladder is strongly associated with frailty in older individuals. Urology 2017; 106: 26-30.

12 Gajewski JB, Schurch B, Hamid R, et al: An International Continence Society (ICS) report on the terminology for adult neurogenic lower urinary tract dysfunction (ANLUTD). Neurourol Urodyn 2018;37:1152-1161.

13 Brading AF: A myogenic basis for the overactive bladder. Urology 1997;50:57-67.

14 de Groat WC: A neurologic basis for the overactive bladder. Urology 1997;50:36-52.

15 Major H, Culligan P, Heit M: Urethral sphincter morphology in women with detrusor instability. Obstet Gynecol 2002;99:63-68.

16 Clobes A, DeLancey JO, Morgan DM: Urethral circular smooth muscle in young and old women. Am J Obstet Gynecol 2008;198:587. e1-5.

17 Yamaguchi O, Nomiya M, Andersson KE: Functional consequences of chronic bladder ischemia. Neurourol Urodyn 2014;33:54-58.

18 Birder LA, Andersson KE, Kanai AJ, HannaMitchell AT, Fry CH: Urothelial mucosal signaling and the overactive bladder-ICI-RS 2013. Neurourol Urodyn 2014;33:597-601.

19 Weiss JP, Blaivas JG, Bliwise DL, et al: The evaluation and treatment of nocturia: a consensus statement. BJU Int 2011;108:6-21.

20 De Nunzio C, Cindolo L, Gacci M, et al: Metabolic syndrome and lower urinary tract symptoms in patients with benign prostatic enlargement: a possible link to storage symptoms. Urology 2014;84:1181-1187.
21 Subak LL, Wing R, West DS, et al: Weight loss to treat urinary incontinence in overweight and obese women. New Engl J Med 2009;360: 481-490.

22 Laycock J: Clinical evaluation of the pelvic floor; in Schussler B LJ, Norton P, Stanton SL (eds): Pelvic Floor Re-education. London, Springer, 1994, pp 42-48.

23 Ayeleke RO, Hay-Smith EJ, Omar MI: Pelvic floor muscle training added to another active treatment versus the same active treatment alone for urinary incontinence in women. Cochrane Database Syst Rev 2015;11:CD010551.

24 Dumoulin C, Hay-Smith EJ, Mac Habee-Seguin G: Pelvic floor muscle training versus no treatment, or inactive control treatments, for urinary incontinence in women. Cochrane Database Syst Rev 2014;5:CD005654.

25 Burgio KL: Update on behavioral and physical therapies for incontinence and overactive bladder: the role of pelvic floor muscle training. Curr Urol Rep 2013;14:457-464.

26 Angelini K: Pelvic floor muscle training to manage overactive bladder and urinary incontinence. Nurs Womens Health 2017;21: 51-57.

27 Hay Smith J BB, Burgio K, Dumoulin C, Hagen $\mathrm{S}$, Moore $\mathrm{K}$, et al: Adult conservative management; in Abrams P, Cardozo L, Khoury S, Wein A (eds): Incontinence, 4th International Consultation on Incontinence. Paris, Health Publications, 2009, pp 11011227.

28 Nygaard IE, Shaw JM: Physical activity and the pelvic floor. Am J Obstet Gynecol 2016; 214:164-171.

29 Andersson KE CL, Cruz F, Lee KS, Sahai A, Wein AJ: Pharmacological treatment of urinary incontinence; in Abrams $\mathrm{P}$, Cardozo L, Wagg A, Wein A (eds): Incontinence, ed 6. Bristol, International Continence Society, 2017. 
30 Batista JE, Kolbl H, Herschorn S, et al: The efficacy and safety of mirabegron compared with solifenacin in overactive bladder patients dissatisfied with previous antimuscarinic treatment due to lack of efficacy: results of a noninferiority, randomized, phase IIIb trial. Ther Adv Urol 2015;7:167-179.

31 Torimoto K, Matsushita C, Yamada A, et al: Clinical efficacy and safety of mirabegron and imidafenacin in women with overactive bladder: a randomized crossover study (the MICRO study). Neurourol Urodyn 2017;36: 1097-1103.

32 Drake MJ, Chapple C, Esen AA, et al: Efficacy and safety of mirabegron add-on therapy to solifenacin in incontinent overactive bladder patients with an inadequate response to initial 4-week solifenacin monotherapy: a randomised double-blind multicentre phase $3 \mathrm{~B}$ study (BESIDE). Eur Urol 2016;70:136-145.
33 Andersson KE: Pharmacology: Cardiovascular effects of mirabegron. Nat Rev Urol 2017; 14:587-588.

34 Tubaro A, Puccini F, De Nunzio C: The management of overactive bladder: percutaneous tibial nerve stimulation, sacral nerve stimulation, or botulinum toxin? Curr Opin Urol 2015;25:305-310.

35 Peters KM, Carrico DJ, Perez-Marrero RA, et al: Randomized trial of percutaneous tibial nerve stimulation versus sham efficacy in the treatment of overactive bladder syndrome: results from the SUmiT trial. J Urol 2010;183: 1438-1443.

36 Peters KM, Macdiarmid SA, Wooldridge LS, et al: Randomized trial of percutaneous tibial nerve stimulation versus extended-release tolterodine: results from the overactive bladder innovative therapy trial. J Urol 2009;182: 1055-1061.
37 Peters KM, Carrico DJ, Wooldridge LS, Miller CJ, MacDiarmid SA: Percutaneous tibial nerve stimulation for the long-term treatment of overactive bladder: 3-year results of the STEP study. J Urol 2013;189:2194-2201.

38 Peeters K, Sahai A, De Ridder D, Van Der Aa F: Long-term follow-up of sacral neuromodulation for lower urinary tract dysfunction. BJU Int 2014;113:789-794.

39 Amundsen CL, Richter HE, Menefee SA, et al: OnabotulinumtoxinA vs sacral neuromodulation on refractory urgency urinary incontinence in women: a randomized clinical trial. Jama 2016;316:1366-1374.

40 Drake MJ, Nitti VW, Ginsberg DA, et al: Comparative assessment of the efficacy of onabotulinumtoxinA and oral therapies (anticholinergics and mirabegron) for overactive bladder: a systematic review and network meta-analysis. BJU Int 2017;120:611-622. 